\title{
Spectra of finitely presented lattice-ordered Abelian groups and MV-algebras, part 2.
}

\author{
Vincenzo Marra ${ }^{1}$, Daniel McNeill ${ }^{2 *}$, and Andrea Pedrini ${ }^{1}$ \\ 1 Dipartimento di Matematica "Federigo Enriques" \\ Università degli Studi di Milano \\ Via Cesare Saldini 50, 20133 Milano, Italy \\ vincenzo.marra@unimi.it, andrea.pedrini@unimi.it \\ 2 Dipartimento di Scienze Teoriche e Applicate \\ Università degli Studi dell'Insubria \\ Via Mazzini, 5, 21100 Varese, Italy \\ danmcne@gmail.com
}

\section{Introduction}

This is the second part of a series of two abstracts, the first being by Andrea Pedrini. For background and notation on lattice-ordered Abelian groups ( $\ell$-groups for short), vector lattices, and $\mathbb{Q}$-vector lattices, and their spectral spaces, please see her submission.

If $G$ is an $\ell$-group (or vector lattice, or $\mathbb{Q}$-vector lattice), write $\operatorname{Spec} G, \operatorname{Max} G$, and $\operatorname{Min} G$ for its prime spectral space with the spectral topology, its subspace of maximal congruences, and its subspace of minimal congruences, respectively. Note then that Spec $G$ is completely normal, meaning that the collection of prime congruences containing any given prime congruence is linearly ordered [1, 2.4.1 and 10.1.11]. Moreover, we always assume that $\operatorname{Spec} G$ is compact. Recall [1, 10.2.2] that this is the case if, and only if, $G$ can be equipped with a (strong order) unit. Under this assumption, $\operatorname{Max} G$ is a compact Hausdorff space [1, 10.2.2], and all compact Hausdorff spaces arise in this manner: the vector lattice $\mathrm{C}(X)$ of all real-valued continuous functions on a compact Hausdorff space $X$ is such that $\operatorname{Max} \mathrm{C}(X)$ is naturally homeomorphic to $X$, by a classical result of Yosida. Hence, $\operatorname{Max} G$ is the most general possible compact Hausdorff space.

There is a surjective function

$$
\lambda: \operatorname{Min} G \rightarrow \operatorname{Max} G
$$

defined by $\lambda(\mathfrak{p}):=\uparrow \mathfrak{p} \cap \operatorname{Max} G$ for each $\mathfrak{p} \in \operatorname{Min} G$. It is known that $\lambda$ is continuous [1, 10.2.5]. We explore the extent to which $\lambda$ may be regarded as an analogue of the absolute of Max $G$. We provide background on the absolute $\mathrm{E}(X)$ of a Hausdorff space $X$ in Section 2 below. We show that in the case of finitely presented structures (see Pedrini's abstract) the analogy is strong. Indeed, $\lambda: \operatorname{Min} G \rightarrow \operatorname{Max} G$ fails to be the absolute of $\operatorname{Max} G$ for the single reason that $\operatorname{Min} G$, though a Boolean space, is not extremally disconnected; see Proposition 4.1. In fact, we prove:

Theorem 1.1. Let $G$ be a finitely presented $\ell$-group (or a vector lattice, or $\mathbb{Q}$-vector lattice). The map $\lambda$ in (1) is a closed, compact, irreducible continuous surjection. Moreover, there exists a unique continuous surjection

$$
h: \mathrm{E}(\operatorname{Max} G) \rightarrow \operatorname{Min} G
$$

*Presenting author. 
that makes the following diagram commute.

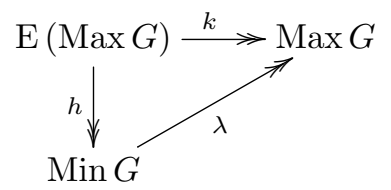

Moreover, the pair $(\mathrm{E}(\operatorname{Max} G), h)$ is the absolute of $\operatorname{Min} G$.

\section{The absolute of a Hausdorff space}

For background on the absolute see [2]. Recall that a topological space is called zero-dimensional if it has a base for the topology consisting only of clopen sets. We call a space extremally disconnected if the closure of every open set is open. Given a map between topological spaces $f: X \rightarrow Y$, we say $f$ is closed if the image of a closed set in $X$ is closed in $Y$, while the map is compact if the fibers of points are compact. Finally, we say $f$ is irreducible if it is a surjection, and there is no proper closed subset $A \subset X$ whose image is all of $Y$.

Given a Hausdorff space $X$, we may construct a space, denoted $\mathrm{E}(X)$, which is:

1. Hausdorff,

2. zero-dimensional, and

3. extremally disconnected,

together with a (surjective) function $k: \mathrm{E}(X) \rightarrow X$ which is:

i. closed,

ii. compact,

iii. irreducible, and

iv. continuous.

The pair $(\mathrm{E}(X), k)$ is called the absolute of the space $X$, and is (essentially) unique with respect to the properties above. That is, if $\left(\mathrm{E}^{\prime}(X), k^{\prime}\right)$ is another pair satisfying the given properties, then there is a homeomorphism $h: \mathrm{E}(X) \rightarrow \mathrm{E}^{\prime}(X)$ so that the following diagram commutes:

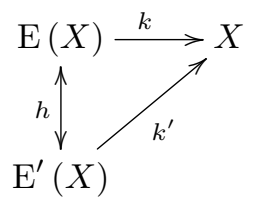

In order to construct $\mathrm{E}(X)$, let $\mathscr{R}(X)$ denote the collection of regular open subsets of $X$, i.e. $U \in \mathscr{R}(X)$ if, and only if, $U=\operatorname{int} \operatorname{cl} U$, where 'int' and 'cl' denote the interior and closure operators associated to the topology of $X$, respectively. It is well known that $\mathscr{R}(X)$ is a complete Boolean algebra under set-theoretic intersections, with joins given by

$$
\bigvee_{i \in I} U_{i}:=\operatorname{int} \mathrm{cl}\left(\bigcup_{i \in I} U_{i}\right),
$$


meets given by

$$
\bigwedge_{i \in I} U_{i}:=\operatorname{int}\left(\bigcap_{i \in I} U_{i}\right)
$$

and negation given by

$$
\neg U:=\operatorname{int} X \backslash U \text {. }
$$

We can then take $\mathrm{E}(X)$ to be the set of convergent maximal filters of $\mathscr{R}(X)$ equipped with the Stone topology. Here, a maximal filter $\mathscr{U}$ of $\mathscr{R}(X)$ is convergent if $\bigcap_{U \in \mathscr{U}} \mathrm{cl} U \neq \emptyset$. When $\mathscr{U}$ is convergent the latter intersection can be shown to be a singleton $\left\{p_{\mathscr{U}}\right\} \subseteq X$. We therefore define the map $k: \mathrm{E}(X) \rightarrow X$ by setting

$$
k(\mathscr{U}):=p_{\mathscr{U}}
$$

for each convergent maximal filter $\mathscr{U}$ of $\mathscr{R}(X)$.

When the space $X$ is, in addition, compact, then $\mathrm{E}(X)$ turns out to be compact, too. Indeed, in this case every maximal filter $\mathscr{U}$ of $\mathscr{R}(X)$ is convergent, and $\mathrm{E}(X)$ is the Stone space of the Boolean algebra $\mathscr{R}(X)$. Every continuous map between compact Hausdorff spaces is automatically closed and compact. We can then drop i and ii from the list above. Also note that a compact Hausdorff extremely disconnected space is automatically zero-dimensional, so 2 above is redundant if one restricts to the compact case.

We next show that Min $G$ may be constructed out of Max $G$ by a procedure that is entirely analogous to the construction of $\mathrm{E}(X)$ out of $X$.

\section{Construction of $\operatorname{Min} G$}

Given any Heyting algebra $H$, set

$$
\mathrm{R}(H):=\{x \in H \mid \exists y \in H \text { with } x=\neg y\} .
$$

Then $\mathrm{R}(H)$ is a Boolean algebra, known as the algebra of regular elements of $H$. If $X$ is a compact Hausdorff space and $\mathscr{O}(X)$ is the Heyting algebra of open sets of $X$, then $\mathrm{R}(\mathscr{O}(X))=$ $\mathscr{R}(X)$. We are going to show that $\operatorname{Min} G$ arises as the Stone space of the Boolean algebra of regular elements in the Heyting algebra of principal congruences of the finitely presented structure $G$.

Throughout the sequel, we write $\cong$ to denote the existence of either a homeomorphism of spaces or an isomorphism of algebraic structures. Let $G$ be a finitely presented $\ell$-group (or a vector lattice, or $\mathbb{Q}$-vector lattice). By the results announced in Pedrini's abstract, we know that the lattice $\mathrm{K}(G)$ of principal congruences on $G$ is a Heyting subalgebra of the Heyting algebra Con $G$ of all congruences on $G$. We prove:

Theorem 3.1. Let $G$ be a finitely presented $\ell$-group (or a vector lattice, or $\mathbb{Q}$-vector lattice). Let $E$ be the Stone space of the Boolean algebra $\mathrm{R}(\mathrm{K}(G))$, then $\operatorname{Min} G \cong E$.

The proof goes through the geometric representation of $G$. In more detail, when $G$ is either an $\ell$-group or a $\mathbb{Q}$-vector lattice, we have the following.

- There is a rational polyhedron $P \subseteq \mathbb{R}^{n}$ such that Max $G \cong P$. 
- The opposite $\left(\operatorname{Sub}_{\mathbb{Q}} P\right)^{\text {op }}$ of the lattice $\operatorname{Sub}_{\mathbb{Q}} P$ of rational polyhedra contained in $P$ is in fact a Heyting algebra, isomorphic to the Heyting algebra $\mathrm{K}(G)$ of principal congruences on $G$.

The crux of the proof is then the observation that $\mathrm{R}(\mathrm{K}(G))$ is canonically identified with the Boolean algebra of open polyhedre ${ }^{1}$ contained in $P$ which are also regular open sets. When $G$ is a vector lattice, then the same construction works using the lattice $\operatorname{Sub} P$ of all polyhedra contained in the representing polyhedron $P$, rather than the lattice of rational polyhedra.

In summary, we see that $\operatorname{Min} G$ is the Stone space of the Boolean algebra of those regular opens in $\operatorname{Max} G$ which are determined by principal congruences ${ }^{2}$

\section{Characterization of the space of minimal primes of finitely presented $\mathbb{Q}$-structures}

In the case of finitely presented $\ell$-groups or $\mathbb{Q}$-vector lattices, it is possible to describe $\operatorname{Min} G$ explicitly:

Proposition 4.1. If $G$ is either a finitely presented $\mathbb{Q}$-vector lattice, or a finitely presented $\ell$-group, then $\operatorname{Min} G$ is homeomorphic to the Cantor space, together with finitely many isolated points.

The proof uses Tarski's classical result that there is a single countable atomless Boolean algebra up to isomorphism, namely, the algebra of clopen sets of the Cantor space (equivalently, the Tarski-Lindenbaum algebra of classical propositional logic over countably many variables, or Brouwer's result that every zero-dimensional compact metric space without isolated points is homeomorphic to the Cantor space). It thus suffices to show that the algebra of clopens of $\operatorname{Min} G$ has these properties, and we do this through a geometric argument.

Remark 4.2. If $G$ is a finitely presented vector lattice, then $\operatorname{Min} G$ is not necessarily homeomorphic to the Cantor space. The problem is that the algebra of clopens of Min $G$ can be uncountable in this case.

The preceding notwithstanding, we have:

Theorem 4.3. If $G$ is a finitely presented $\ell$-group (or vector lattice, or $\mathbb{Q}$-vector lattice),

$$
\mathrm{E}(\operatorname{Max} G) \cong \mathrm{E}(\operatorname{Min} G) .
$$

To prove this we use standard results from 2 . In case $G$ is either a finitely presented $\ell$-group or $\mathbb{Q}$-vector lattice, $\operatorname{Min} G$ and $\operatorname{Max} G$ are both compact metric spaces, and the result follows. When $G$ is a vector lattice, $\operatorname{Min} G$ need not be metrizable (compare Remark 4.2). However, Min $G$ has a countable $\pi$-basis, that is, a countable collection $\mathfrak{B}$ of open sets such that for any open set $U$ of $\operatorname{Min} G$ there is some $B \in \mathfrak{B}$ with $B \subseteq U$. This entails that $\mathrm{E}$ (Min $G$ ) is the absolute of the Cantor space whenever $\operatorname{Min} G$ has no isolated points. From this the theorem follows easily.

\footnotetext{
${ }^{1}$ We call a polyhedron $Q \subseteq P$ open if its complement $P \backslash Q$ in $P$ is a polyhedron.

${ }^{2}$ The fact that Min $G$ is a Boolean space is also stated as Corollary 5.1 in Pedrini's abstract; Theorem 3.1 proves more.
} 


\section{Proof of Theorem 1.1}

Lemma 5.1. Let $G$ be a finitely presented $\ell$-group (or a vector lattice, or $\mathbb{Q}$-vector lattice). Consider the map $\lambda: \operatorname{Min} G \rightarrow \operatorname{Max} G$ as in (1), then $\lambda$ is closed, compact, irreducible, continuous and onto.

The last two properties are known, as mentioned in the Introduction. Compactness amounts to an application of Corollary 5.2 in Pedrini's abstract. The remaining two properties are proved using the geometric representation of $G$ recalled in Section 3 of Pedrini's abstract.

End of Proof of Theorem 1.1. In light of Lemma 5.1 we only need prove the 'Moreover' statement. By Theorem 4.3 we have $\mathrm{E}(\operatorname{Min} G) \cong \mathrm{E}(\operatorname{Max} G)$, so that the pair $\left(\mathrm{E}(\operatorname{Max} G), k_{\min }\right)$ is the absolute space of $\operatorname{Min} G$ with covering map $k_{\min }: \mathrm{E}(\operatorname{Max} G) \rightarrow \operatorname{Min} G$. We first show that $k_{\min }$ commutes the diagram above, i.e. $k=\lambda \circ k_{\min }$. Set $k^{\prime}:=\lambda \circ k_{\min }$ for short. It is obvious that $k^{\prime}$ is onto, continuous, and closed. It is also compact. Indeed, if $p \in \operatorname{Max} G$ then $\lambda^{-1}(p)$ is compact because $\lambda$ is compact, hence closed because $\operatorname{Min} G$ is compact Hausdorff. Hence $\left(k^{\prime}\right)^{-1}(p)$ is closed and therefore compact, because $\mathrm{E}(\operatorname{Max} G)$ is compact Hausdorff. Moreover, $k^{\prime}$ is irreducible. To see this, note that If $C \subset \mathrm{E}(\operatorname{Max} G)$ is closed then $k_{\min }(C)$ is a proper closed subset of $\operatorname{Min}(G)$, because $k_{\min }$ is irreducible and closed. Hence $k^{\prime}(C) \neq \operatorname{Max} G$, because $\lambda$ is irreducible. This shows that $\left(\mathrm{E}(\operatorname{Max} G), k^{\prime}\right)$ is the absolute of $\operatorname{Max} G$, and therefore $k^{\prime}=k$ by its uniqueness property. Hence $k_{\min }$ commutes the diagram above.

It remains to show that any $h: \operatorname{E}(\operatorname{Max} G) \rightarrow \operatorname{Min} G$ as in the statement agrees with $k_{\min }$. For this, note that $h$ is closed: if $C \subseteq \mathrm{E}(\operatorname{Max} G)$ is closed, then $k(C)$ is closed because $k$ is closed, and $\lambda^{-1}(k(C))$ is closed by continuity. Moreover, $h$ is compact: if $p \in \operatorname{Min} G$ then $k^{-1}(\lambda(p))$ is compact because $k$ is compact. Finally, $h$ is irreducible: if $C \subset \mathrm{E}(\operatorname{Max} G)$ satisfies $h(C)=\operatorname{Min} G$ by way of contradiction, then $\lambda(h(C))=k(C)=\operatorname{Max} G$, against the fact that $k$ is irreducible. The theorem is proved.

\section{References}

[1] Alain Bigard, Klaus Keimel, and Samuel Wolfenstein. Groupes et anneaux réticulés. Lecture Notes in Mathematics, Vol. 608. Springer-Verlag, Berlin, 1977.

[2] Jack R. Porter and R. Grant Woods. Extensions and absolutes of Hausdorff spaces. Springer-Verlag, New York, 1988. 